TUM-HEP-580/05

\title{
Future Precision Neutrino Oscillation Experiments and Theoretical Implications*
}

\author{
M. Lindner ${ }^{\dagger}$, \\ Physik Department, Technische Universität München, D-85748 Garching bei München, Germany
}

\begin{abstract}
Future neutrino oscillation experiments will lead to precision measurements of neutrino mass splittings and mixings. The flavour structure of the lepton sector will therefore at some point become better known than that of the quark sector. This article discusses the potential of future oscillation experiments on the basis of detailed simulations with an emphasis on experiments which can be done in about ten years. In addition, some theoretical implications for neutrino mass models will be briefly discussed.
\end{abstract}

PACS numbers: 12.15.Ff, 14.60.Lm, 14.60.Pq, 14.60.St

\section{Introduction}

The observation of atmospheric neutrino oscillations by the SuperKamiokande experiment [1] triggered a remarkable discovery phase. The initial evidence turned into a solid proof of neutrino flavour conversions as well as of the $\mathrm{L} / \mathrm{E}$ dependence as required by oscillations. The solar neutrino problem has also been resolved in the last years. The Gallex experiment 2] detected initially a rate effect which implied flavour conversion on the basis of solar models. The SNO experiment proved then model independent neutrino flavour transitions [3, 4]. The initially allowed parameter islands were cleaned up by the KamLAND experiment, which demonstrated finally with reactor anti-neutrinos [5] that the so-called LMA-solution is correct. Altogether the existing experimental results fit now very nicely into a picture with three massive neutrinos, which corresponds to the simplest scenario for three generations. The only exemption is the disputed LSND result [7, which would have far reaching consequences if it were confirmed, but this possibility will be ignored here. The oscillations of three neutrino generations involve then two mass-squared differences $\Delta m_{12}^{2} \simeq \Delta m_{\text {sol. }}^{2}$ and $\Delta m_{23}^{2} \simeq \Delta m_{\text {atm. }}^{2}$, three mixing angles, $\theta_{12}, \theta_{23}$, and $\theta_{13}$, and a CP-violating phase $\delta$. Atmospheric neutrino data [8] and the first results from the $\mathrm{K} 2 \mathrm{~K}$ long-baseline accelerator experiment [8] determine $\Delta m_{23}^{2}=\left(2.2_{-0.4}^{+0.6}\right) \times 10^{-3} \mathrm{eV}^{2}$ and $\theta_{23} \approx 45^{\circ}$ [8, 9], whereas solar neutrino data [10, 11, combined with the results from the KamLAND reactor experiment [5] lead to $\Delta m_{12}^{2}=\left(8.2_{-0.3}^{+0.3}\right) \times 10^{-5} \mathrm{eV}^{2}$ and $\tan ^{2} \theta_{12}=0.39_{-0.04}^{+0.05}[$. The results can now approximatively be summarized by two independent two flavour oscillations where the solar and atmospheric $\Delta m^{2}$ values are roughly now known.

The key parameter for genuine three flavour effects is the mixing angle $\theta_{13}$ which is so far only known to be small from the CHOOZ [12, 13] and Palo Verde [14] experiments. The current bound for $\theta_{13}$ depends on the

\footnotetext{
*Talk presented at the Nobel Symposium 129: Neutrino Physics, Enköping, Sweden, 2004

${ }^{\dagger}$ Email address: lindner@ph.tum.de
} 
value of the atmospheric mass squared difference and it gets rather weak for $\Delta m_{31}^{2} \lesssim 2 \times 10^{-3} \mathrm{eV}^{2}$. However, in that region an additional constraint on $\theta_{13}$ from global solar neutrino data becomes important [15]. At the current best fit value of $\Delta m_{31}^{2}=2.2 \times 10^{-3} \mathrm{eV}^{2}$ we have at $3 \sigma$ the bound $\sin ^{2} \theta_{13} \leq 0.041$ [9]. There is no reason why $\theta_{13}$ should vanish and one should expect therefore $\theta_{13}$ to be finite.

One might think that neutrino oscillations are in future less interesting, since it will lead only to parameter improvements of the leading $2 \times 2$ oscillations and maybe a finite parameter value of $\theta_{13}$. However, such a view misses completely the fact that the neutrino sector is, unlike the quark sector, not obscured by hadronic uncertainties. The precision to which the underlying flavour information is determined will therefore only be limited by the ultimate experimental precision. If high precision measurements are possible, then they will be very sensitive tests of flavour models and related topics, like the unitarity of three flavours. Genuine three flavour oscillation effects occur only for a finite value of $\theta_{13}$ and establishing a finite value of $\theta_{13}$ is therefore one of the next milestones in neutrino physics. Leptonic CP violation is another three flavour effect which can only be tested if $\theta_{13}$ is finite. The usual see-saw scenario includes besides $\delta$ in addition two further Majorana CP phases in the light neutrino sector, as well as other CP phases in the heavy Majorana sector, which are involved in leptogenesis. In general the heavy and light CP phases are not connected, but most flavour models create relations between these two sectors, relating thus low energy leptonic $\mathrm{CP}$ violation to leptogenesis and mass models. Precision measurements of neutrino oscillations test therefore very interesting questions of particle physics which are connected to the origin of flavour and to phenomenological consequences of flavour. There is thus a very strong motivation to establish first in the next generation of experiments a finite value of $\theta_{13}$ in order to aim in the long run at a measurement of leptonic CP violation [16, 17, 18, 19, 20].

\section{Three neutrino oscillation in matter}

An effective two flavour treatment is insufficient for future oscillation experiments and matter effects must be included in addition. The generalization of the oscillation formulae in vacuum to $N$ neutrinos leads to the probabilities for flavour transitions $\nu_{f_{l}} \rightarrow \nu_{f_{m}}$ given by

$$
P\left(\nu_{f_{l}} \rightarrow \nu_{f_{m}}\right)=\underbrace{\delta_{l m}-4 \sum_{i>j} \operatorname{Re} J_{i j}^{f_{l} f_{m}} \sin ^{2} \Delta_{i j}}_{P_{C P}} \underbrace{-2 \sum_{i>j} \operatorname{Im} J_{i j}^{f_{l} f_{m}} \sin 2 \Delta_{i j}}_{P_{\varnothing P}}
$$

where the shorthands $J_{i j}^{f_{l} f_{m}}:=U_{l i} U_{l j}^{*} U_{m i}^{*} U_{m j}$ and $\Delta_{i j}:=\frac{\Delta m_{i j}^{2} L}{4 E}$ have been used. These generalized vacuum transition probabilities depend on all combinations of quadratic mass differences $\Delta m_{i j}^{2}=m_{i}^{2}-m_{j}^{2}$ as well as on different products of elements of the leptonic mixing matrix $U$. We will assume a three neutrino framework, i.e. $1 \leq i, j \leq 3$ and $U$ is a $3 \times 3$ mixing matrix parameterized in the standard way

$$
U=\left(\begin{array}{ccc}
c_{12} c_{13} & s_{12} c_{13} & s_{13} e^{-i \delta} \\
-s_{12} c_{23}-c_{12} s_{23} s_{13} e^{i \delta} & c_{12} c_{23}-s_{12} s_{23} s_{13} e^{i \delta} & s_{23} c_{13} \\
s_{12} s_{23}-c_{12} c_{23} s_{13} e^{i \delta} & -c_{12} s_{23}-s_{12} c_{23} s_{13} e^{i \delta} & c_{23} c_{13}
\end{array}\right),
$$

where $c_{i j}=\cos \left(\theta_{i j}\right)$ and $s_{i j}=\sin \left(\theta_{i j}\right) . U$ contains three leptonic mixing angles and one Dirac-like leptonic $\mathrm{CP}$ phase $\delta$. Note that the most general mixing matrix for three Majorana neutrinos contains two further 
Majorana-like CP phases, but it can easily be seen that these extra phases do not enter in the above oscillation formulae. Disappearance probabilities, i.e. the transitions $\nu_{f_{l}} \rightarrow \nu_{f_{l}}$, do not even depend on $\delta$, since $J_{i j}^{f_{l} f_{l}}$ is only a function of the modulus of elements of $U$. Appearance probabilities, like $\nu_{e} \rightarrow \nu_{\mu}$ are therefore the place where leptonic CP violation can be studied. Eq. (11) contains a CP conserving part $P_{C P}$ and a CP violating part $P_{\mathscr{C P}}$, and both terms depend on the CP phase $\delta$. An obvious extraction strategy for CP-violation would thus be to look at CP asymmetries [21. Note, however, that the beams of a long baseline experiment traverse the Earth and the presence of matter violates CP by itself. This implies modifications of eq. (11) and it makes a measurement of leptonic CP violation more involved.

The general oscillation formulae in vacuum, eq. (1), lead to well known, but rather lengthy trigonometric expressions for the oscillation probabilities in vacuum. These expressions become even longer and do not exist in closed form when arbitrary matter corrections are taken into account. For effectively constant matter densities, which is often a good assumption, the problem simplifies somewhat, but the general oscillation probabilities are still very lengthy. The Hamiltonian describing three neutrino oscillation in matter can then be written in flavour basis as

$$
H=\frac{1}{2 E_{\nu}} U\left(\begin{array}{ccc}
m_{1}^{2} & 0 & 0 \\
0 & m_{2}^{2} & 0 \\
0 & 0 & m_{3}^{2}
\end{array}\right) U^{T}+\frac{1}{2 E_{\nu}}\left(\begin{array}{ccc}
A+A^{\prime} & 0 & 0 \\
0 & A^{\prime} & 0 \\
0 & 0 & A^{\prime}
\end{array}\right) .
$$

The first term describes oscillations in vacuum in flavour basis. The quantities $A$ and $A^{\prime}$ in the second term are given by the charged current and neutral current contributions to coherent forward scattering in matter. The charged current contribution is given by

$$
A= \pm \frac{2 \sqrt{2} G_{F} Y \rho E_{\nu}}{m_{n}}=2 V E_{\nu}
$$

where $G_{F}$ is Fermi's constant, $Y$ is the number of electrons per nucleon, $m_{n}$ is the nucleon mass and $\rho$ is the matter density. A is positive for neutrinos in matter and anti-neutrinos in anti-matter, while it is negative for anti-neutrinos in matter and neutrinos in anti-matter. The flavour universal neutral current contributions $A^{\prime}$ lead to an overall phase which does not enter the transition probabilities. The over-all neutrino mass scale $m_{1}^{2}$ can be written as a term proportional to the unit matrix and can similarly be removed, such that only $\Delta m_{21}^{2}$ and $\Delta m_{31}^{2}$ remain in the first term of eq. (3). After re-diagonalization of the Hamiltonian in constant matter density one finds that matter effects lead in a very good approximation to an $A$-dependent parameter mapping in the 1-3 subspace which can be written as [22]

$$
\begin{aligned}
\sin ^{2} 2 \theta_{13, m} & =\frac{\sin ^{2} 2 \theta_{13}}{C_{ \pm}^{2}}, \\
\Delta m_{31, m}^{2} & =\Delta m_{31}^{2} C_{ \pm}, \\
\Delta m_{32, m}^{2} & =\frac{\Delta m_{31}^{2}\left(C_{ \pm}+1\right)+A}{2} \\
\Delta m_{21, m}^{2} & =\frac{\Delta m_{31}^{2}\left(C_{ \pm}-1\right)-A}{2} .
\end{aligned}
$$

The index $m$ denotes effective quantities in matter, where

$$
C_{ \pm}^{2}=\left(\frac{A}{\Delta m_{31}^{2}}-\cos 2 \theta\right)^{2}+\sin ^{2} 2 \theta
$$


Note that $A$ in $C_{ \pm}$can change its sign and the mappings for neutrinos and anti-neutrinos are therefore different, resulting in different effective mixings and masses. This is an important effect, which will allow detailed tests of coherent forward scattering of neutrinos in matter. Note that oscillations in matter depend unlike vacuum oscillations on the sign of $\Delta m_{31}^{2}$. This allows to extract the $\operatorname{sign}\left(\Delta m_{31}^{2}\right)$ via matter effects.

Inserting the parameter mappings eqs. (5)-(8) into the full oscillation formulae leads still to quite lengthy expressions for the oscillation probabilities in matter, where it is not easy to oversee all effects. It is therefore instructive to simplify the problem further such that a qualitative analytic understanding of all effects becomes possible, while quantitative statements should be evaluated numerically with the full expressions. The key for further simplification is to expand the oscillation probabilities in small quantities. These expansion parameters are $\alpha=\Delta m_{21}^{2} / \Delta m_{31}^{2}=\mathrm{O}\left(10^{-2}\right)$ and $\sin ^{2} 2 \theta_{13} \leq 0.16$. The matter effects can be parameterized by the dimensionless quantity $\hat{A}=A / \Delta m_{31}^{2}=2 V E / \Delta m_{31}^{2}$, where $V=\sqrt{2} G_{F} n_{e}$. The oscillation probabilities for all channels can in this way be significantly simplified [23]. Using $\Delta \equiv \Delta_{31}$, the leading terms for $P\left(\nu_{\mu} \rightarrow \nu_{\mu}\right)$ and $P\left(\nu_{e} \rightarrow \nu_{\mu}\right)$ can, for example, be written as [17, 24, 22]

$$
\begin{aligned}
& P\left(\nu_{\mu} \rightarrow \nu_{\mu}\right) \approx \\
& \quad 1-\cos ^{2} \theta_{13} \sin ^{2} 2 \theta_{23} \sin ^{2} \Delta+2 \alpha \cos ^{2} \theta_{13} \cos ^{2} \theta_{12} \sin ^{2} 2 \theta_{23} \Delta \cos \Delta, \\
& P\left(\nu_{e} \rightarrow \nu_{\mu}\right) \approx \sin ^{2} 2 \theta_{13} \sin ^{2} \theta_{23} \frac{\sin ^{2}((1-\hat{A}) \Delta)}{(1-\hat{A})^{2}} \\
& \quad \pm \sin \delta \cdot \sin 2 \theta_{13} \alpha \sin 2 \theta_{12} \cos \theta_{13} \sin 2 \theta_{23} \sin (\Delta) \frac{\sin (\hat{A} \Delta) \sin ((1-\hat{A}) \Delta)}{\hat{A}(1-\hat{A})} \\
& \quad+\cos \delta \cdot \sin 2 \theta_{13} \alpha \sin 2 \theta_{12} \cos \theta_{13} \sin 2 \theta_{23} \cos (\Delta) \frac{\sin (\hat{A} \Delta) \sin ((1-\hat{A}) \Delta)}{\hat{A}(1-\hat{A})} \\
& \quad+\alpha^{2} \sin ^{2} 2 \theta_{12} \cos ^{2} \theta_{23} \frac{\sin ^{2}(\hat{A} \Delta)}{\hat{A}^{2}},
\end{aligned}
$$

where "+" in eq. (11) stands for neutrinos and "-" for anti-neutrinos. The most important feature of eq. (11) is that all interesting effects in the $\nu_{e} \rightarrow \nu_{\mu}$ transition depend crucially on $\theta_{13}$. The size of $\sin ^{2} 2 \theta_{13}$ determines thus if the total transition rate, matter effects, effects due to the sign of $\Delta m_{31}^{2}$ and $\mathrm{CP}$ violating effects are measurable. This is the reason why the size of $\theta_{13}$ is one of the most important questions for future oscillation experiments.

Before we discuss some features of eqs. (10) and (11) in more detail, we would like to comment on the underlying assumptions and the reliability of these equations. First eqs. (10) and (11) are an expansion in terms of the small quantities $\alpha$ and $\sin 2 \theta_{13}$. Higher order terms are suppressed at least by another power of one of these small parameters and these corrections are thus typically at the percent level. Note that the expansion in $\alpha$ is actually an expansion in the solar and not the atmospheric frequency. The expansion does therefore not break down at the first atmospheric oscillation maximum, i.e. at $\Delta \simeq 1$, but at much larger baselines before the first (sub-dominant) solar oscillation maximum, i.e. at $\alpha \Delta \simeq 1$. The latter condition gives an upper bound for the baseline where eqs. (10) and (11) are good approximations

$$
L \lesssim 8000 \mathrm{~km}\left(\frac{E_{\nu}}{\mathrm{GeV}}\right)\left(\frac{10^{-4} \mathrm{eV}^{2}}{\Delta m_{21}^{2}}\right)
$$


while the first oscillation maximum sits at $\alpha \cdot L \simeq L / 30$. Eqs. (10) and (11) are therefore excellent approximations at and well beyond the first oscillation maximum of long baseline experiments. The matter corrections in eqs. (10) and (11) are derived for constant average matter density which is a good approximation.

Note that all quantitative results which will be presented are based on numerical simulations in matter. The results are therefore not affected by any approximation. Eqs. (10) and (11) will only be used to understand the problem analytically, which is extremely helpful in order to oversee the multi-dimensional parameter space.

In addition to long baseline experiments, reactor experiments with identical near and far detectors have an excellent potential for precise measurements. The near detector is used to eliminate many common systematical errors and the far detector is located typically at a baseline of a few kilometer. For these short baselines matter effects can be ignored and one finds to second order in the small quantities $\sin 2 \theta_{13}$ and $\alpha$ for the oscillation probability

$$
1-P_{\bar{e} \bar{e}}=\sin ^{2} 2 \theta_{13} \sin ^{2} \Delta_{31}+\alpha^{2} \Delta_{31}^{2} \cos ^{4} \theta_{13} \sin ^{2} 2 \theta_{12} .
$$

At the first atmospheric oscillation maximum, $\Delta_{31}$ is approximately $\pi / 2$ and $\sin ^{2} \Delta_{31}$ is close to one, which means that the second term on the right-hand side of this equation can be neglected for $\sin ^{2} 2 \theta_{13} \gtrsim 10^{-3}$. The reactor measurement is dominated in this case at short baselines by the product of $\sin ^{2} 2 \theta_{13}$ and $\sin ^{2} \Delta_{31}$, which must be measured as deviation from one. Eq. (13) implies that correlations and degeneracies play essentially no role in reactor experiments. The behavior in the $\sin ^{2} 2 \theta_{13}-\Delta m_{21}^{2}$-plane will also be different since eq. (13) is essentially independent of $\Delta m_{21}^{2}$. A reactor experiment will improve the global parameter determination in two ways. First, a direct, essentially uncorrelated and clean measurement for $\theta_{13}$ [25] can be obtained which can be used to disentangle the long baseline results. Secondly, the reactor measurement can replace the cross-section suppressed anti-neutrino running of the accelerator experiments, leading to statistical improvements in the neutrino measurements [26].

\section{Correlations and degeneracies}

Eqs. (10) and (11) exhibit certain parameter correlations and degeneracies, which play an important role in the analysis of long baseline experiments, and which would be hard to understand in a purely numerical analysis of the high dimensional parameter space. The most important properties are:

- Eqs. (10) and (11) depend only on the product $\alpha \cdot \sin 2 \theta_{12}$ or equivalently $\Delta m_{21}^{2} \cdot \sin 2 \theta_{12}$. This are the parameters related to solar oscillations which will be taken as external input. Note that the product is better determined than the product of the individual measurements of $\Delta m_{21}^{2}$ and $\sin 2 \theta_{12}$.

- Next we observe in eq. (11) that the second and third term contain both a factor $\sin (\hat{A} \Delta)$, while the last term contains a factor $\sin ^{2}(\hat{A} \Delta)$. Since $\hat{A} \Delta=V L / 2$, we find that these factors depend only on $L$, resulting in a "magic baseline" 27] when $V L_{\text {magic }}=2 \pi$, where $\sin (\hat{A} \Delta)$ vanishes. At this magic baseline only the first term in eq. (11) survives and $P\left(\nu_{e} \rightarrow \nu_{\mu}\right)$ does no longer depend on $\delta$, $\alpha$ and $\sin 2 \theta_{12}$. This is in principle very important, since it implies that $\sin ^{2} 2 \theta_{13}$ can be determined at the 
magic baseline from the first term of eq. (11) whatever the values and errors of $\delta, \alpha$ and $\sin 2 \theta_{12}$ are. For the given matter density of the Earth we find $L_{\text {magic }}=2 \pi / V \simeq 8100 \mathrm{~km}$ which fits nicely into the Earth. This value is quite amazing, since $V$ is given in terms of completely unrelated constants of nature like $G_{F}$.

- Next we observe that only the second and third term of eq. (11) depend on the CP phase $\delta$, and both terms contain a factor $\sin 2 \theta_{13} \cdot \alpha$, while the first and fourth term of eq. (I1) do not depend on the CP phase $\delta$ and contain factors of $\sin ^{2} 2 \theta_{13}$ and $\alpha^{2}$, respectively. The extraction of CP violation is thus always suppressed by the product $\sin 2 \theta_{13} \cdot \alpha$ and the CP violating terms are obscured by large $\mathrm{CP}$ independent terms if either $\sin ^{2} 2 \theta_{13} \ll \alpha^{2}$ or $\sin ^{2} 2 \theta_{13} \gg \alpha^{2}$. The relative contribution of the CP phase $\delta$ to the probability is thus largest for $\sin ^{2} 2 \theta_{13} \simeq 4 \theta_{13}^{2} \simeq \alpha^{2}$.

- Another observation is that the last term in eq. (11), which is proportional to $\alpha^{2}=\left(\Delta m_{21}^{2}\right)^{2} /\left(\Delta m_{31}^{2}\right)^{2}$, dominates in the limit of tiny $\sin ^{2} 2 \theta_{13}$. The error of $\Delta m_{21}^{2}$ limits therefore for small $\sin ^{2} 2 \theta_{13}$ the parameter extraction. This last term implies a finite transition probability even for $\theta_{13}=0$. Observing $\nu_{e} \rightarrow \nu_{\mu}$ or $\nu_{\mu} \rightarrow \nu_{e}$ appearance transitions does therefore not necessarily establish a finite value of $\theta_{13}=0$ in a three flavour framework.

- Eqs. (10) and (11) suggest that transformations exist which leave these equations invariant. Therefore degeneracies, i.e. parameter sets having identical oscillation probabilities for a fixed $L / E$ are expected. An example of such an invariance is given by a simultaneous replacement of neutrinos by anti-neutrinos and $\Delta m_{31}^{2} \rightarrow-\Delta m_{31}^{2}$. This is equivalent to changing the sign of the second term of eq. (11) and replacing $\alpha \rightarrow-\alpha$ and $\Delta \rightarrow-\Delta$, while $\hat{A} \rightarrow \hat{A}$. It is easy to see that eqs. (10) and (11) are unchanged, but this is not a degeneracy, since neutrinos and anti-neutrinos can be distinguished experimentally.

- The first real degeneracy [28, 29] can be seen in the disappearance probability eq. (10), which is invariant under the replacement $\theta_{23} \rightarrow \pi / 2-\theta_{23}$. Note that the second and third term in eq. (11) are not really invariant under this transformation, but this change in the sub-leading appearance probability can approximately be compensated by tiny parameter shifts. This implies that the degeneracy can in principle be lifted with high precision measurements in the disappearance channels.

- The second degeneracy can be found in the appearance probability eq. (11) in the $\left(\delta-\theta_{13}\right)$-plane [30, 31]. In terms of $\theta_{13}$ (which is small) and $\delta$ the four terms of eq. (11) have the structure

$$
P\left(\nu_{e} \rightarrow \nu_{\mu}\right) \approx \theta_{13}^{2} \cdot F_{1}+\theta_{13} \cdot\left( \pm \sin \delta F_{2}+\cos \delta F_{3}\right)+F_{4}
$$

where the quantities $F_{i}, i=1, . ., 4$ contain all the other parameters. The requirement $P\left(\nu_{e} \rightarrow \nu_{\mu}\right)=$ const. leads for both neutrinos and anti-neutrinos to parameter manifolds of degenerate or correlated solutions. Having both neutrino and anti-neutrino beams, the two channels can be used independently, which is equivalent to considering simultaneously eq. (14) for $F_{2} \equiv 0$ and $F_{3} \equiv 0$. The requirement that these probabilities are now independently constant, i.e. $P\left(\nu_{e} \rightarrow \nu_{\mu}\right)=$ const. for $F_{2} \equiv 0$ and $F_{3} \equiv 0$, leads to more constraint manifolds in the $\left(\delta-\theta_{13}\right)$-plane, but some degeneracies still survive. 
- The third degeneracy [32 is given by the fact that a change in sign of $\Delta m_{31}^{2}$ can essentially be compensated by an offset in $\delta$. Therefore we note again that the transformation $\Delta m_{31}^{2} \rightarrow-\Delta m_{31}^{2}$ leads to $\alpha \rightarrow-\alpha, \Delta \rightarrow-\Delta$ and $\hat{A} \rightarrow-\hat{A}$. All terms of the disappearance probability, eq. (10), are invariant under this transformation. The first and fourth term in the appearance probability eq. (10), which do not depend on the CP phase $\delta$, are also invariant. The second and third term of eq. (10) depend on the CP phase and change by the transformation $\Delta m_{31}^{2} \rightarrow-\Delta m_{31}^{2}$. The fact that these changes can be compensated by an offset in the CP phase $\delta$ is the third degeneracy.

- Altogether there exists an eight-fold degeneracy [29], as long as only the $\nu_{\mu} \rightarrow \nu_{\mu}, \bar{\nu}_{\mu} \rightarrow \bar{\nu}_{\mu}, \nu_{e} \rightarrow \nu_{\mu}$ and $\bar{\nu}_{e} \rightarrow \bar{\nu}_{\mu}$ channels and one fixed $L / E$ are considered. However, eqs. (10) and (11) also imply that the degeneracies can be broken by using in a suitable way information from different $L / E$ values. This can be achieved in total event rates by changing or combining different $L$ or $E$ [33, 34, 35, but it can in principle also be done by using information in the event rate spectrum of a single baseline $L$, which requires detectors with very good energy resolution [17. Another strategy to break the degeneracies is to include further oscillation channels in the analysis ("silver channels") [33, 36].

The discussion of this section shows the strength of the analytic approximations, which allow to understand the complicated parameter interdependence. It also helps to optimally plan experimental setups and to find strategies to resolve the degeneracies.

\section{The potential of future neutrino oscillation experiments}

Triggered by the spectacular results in neutrino physics during the last ten years, several new experimental projects are under way in this field. It is therefore interesting to investigate where we should stand in the determination of neutrino oscillation parameters in ten years from now. It is also interesting to look further and to estimate the ultimate precision which could be obtained.

The precision of quantities like $\sin ^{2} 2 \theta_{13}$ which is found form the simulation of experiments will be presented in a way shown in fig. 1 The bands show how the initial value, which is given by statistics alone (left edge of blue/dark grey band) deteriorates by systematic errors, by parameter correlations (e.g. with the unknown or partly known CP phase) and parameter degeneracies (due to trigonometric ambiguities). It is important to note that a given experiment (or combination of experiments) typically measures some parameter combination with a precision which is considerably better than the final limit. This precision of the experiment is shown in fig. [1 as the right edge of the blue/dark grey band. This precision might be called $\left(\sin ^{2} 2 \theta_{13}\right)_{e f f}$, since it expresses the precision if all other unknown parameters are fixed and no errors are included. However, if one properly extracts a limit of $\sin ^{2} 2 \theta_{13}$ with all unknowns properly taken into account, then one ends up at the right edge of the yellow/light grey band. Distinguishing in this way between the precision and the sensitivity is quite useful, since it also shows the room for improvement by combinations with other similarly precise experiments with other parameter dependence. 


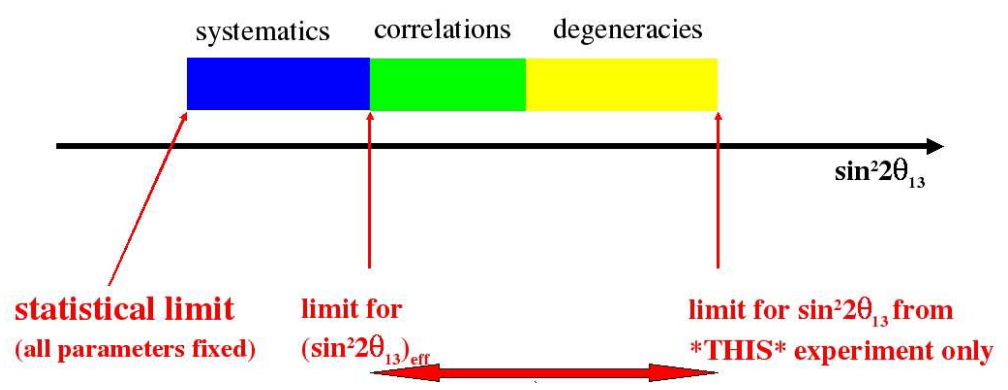

Figure 1: The precision for $\sin ^{2} 2 \theta_{13}$ is shown in colored bands, where the left edge of the blue/dark grey band shows the initial value which is obtained if only statistics is considered. The right edge of the blue/dark grey band is the result after the systematic errors are included. This is the principal precision of the experiment. However, the sensitivity for $\sin ^{2} 2 \theta_{13}$ deteriorates further due to parameter correlations and parameter degeneracies. The final value is the right edge of the yellow/light grey band. The range covered by the green and yellow bands can lead to remarkable synergies when this experiment is combined with another experiment with similar precision, but different parameter dependence.

\subsection{Next generation experiments}

Future oscillation experiments can be grouped according to their time scale of operation. The K2K experiment is already running and it tests the leading atmospheric oscillation already now. Next come the MINOS and CNGS projects which are already operating or under construction, respectively. Therefore we include in our study the conventional beam experiments MINOS [37, and the CNGS experiments ICARUS [38] and OPERA [39. We include also the subsequent superbeam experiments J-PARC to SuperKamiokande (T2K) 40] and NuMI off-axis $(\mathrm{NO} \nu \mathrm{A})$ [41, as well as new reactor neutrino experiments 42] with a near and far detector. The main characteristics of these experiments are summarized in tab. 1 For the reactor experiments we use the Double-CHOOZ proposal (D-CHOOZ) [4] as initial stage setup with roughly $6 \times 10^{4}$ events, and an optimized setup called Reactor-II, with a slightly longer baseline and $6 \times 10^{5}$ events. Such a configuration could be realized at several other sites under discussion [42. The results presented in the following are based on Ref. 44, where more details on the analysis can be found. The simulations of the experiments as well as the statistical analysis is performed with the GLoBES software package [4].

\begin{tabular}{lcccl}
\hline Label & $L[\mathrm{~km}]$ & \multicolumn{4}{c}{$\left\langle E_{\nu}\right\rangle$} & $t_{\text {run }}$ & channel \\
\hline \multicolumn{4}{l}{ Conventional beam experiments: } \\
MINOS & 735 & $3 \mathrm{GeV}$ & $5 \mathrm{yr}$ & $\nu_{\mu} \rightarrow \nu_{\mu, e}$ \\
ICARUS & 732 & $17 \mathrm{GeV}$ & $5 \mathrm{yr}$ & $\nu_{\mu} \rightarrow \nu_{e, \mu, \tau}$ \\
OPERA & 732 & $17 \mathrm{GeV}$ & $5 \mathrm{yr}$ & $\nu_{\mu} \rightarrow \nu_{e, \mu, \tau}$ \\
Off-axis superbeams: & & \\
T2K & 295 & $0.76 \mathrm{GeV}$ & $5 \mathrm{yr}$ & $\nu_{\mu} \rightarrow \nu_{e, \mu}$ \\
NO $\nu \mathrm{A}$ & 812 & $2.22 \mathrm{GeV}$ & $5 \mathrm{yr}$ & $\nu_{\mu} \rightarrow \nu_{e, \mu}$ \\
Reactor experiments: & & \\
D-CHOOZ & 1.05 & $\sim 4 \mathrm{MeV}$ & $3 \mathrm{yr}$ & $\nu_{e} \rightarrow \nu_{e}$ \\
Reactor-II & 1.70 & $\sim 4 \mathrm{MeV}$ & $5 \mathrm{yr}$ & $\nu_{e} \rightarrow \nu_{e}$ \\
\hline
\end{tabular}

Table 1: Characteristics of the considered experiments. 
A first interesting question concerns improvements of $\Delta m_{31}^{2}$ and $\sin ^{2} \theta_{23}$. In tab. [2 we show the precision which can be obtained in the future in comparison to the current precision, as obtained from a global fit to SuperKamiokande (SK) atmospheric and K2K long-baseline data [15. The last row is the precision which can be obtained by combining all experiments. We observe from these numbers, that the accuracy on $\Delta m_{31}^{2}$ can be improved by one order of magnitude, whereas the accuracy on $\sin ^{2} \theta_{23}$ will be improved only by a factor two.

\begin{tabular}{lrr}
\hline & $\left|\Delta m_{13}^{2}\right|$ & $\sin ^{2} \theta_{23}$ \\
\hline current & $88 \%$ & $79 \%$ \\
\hline MINOS+CNGS & $26 \%$ & $78 \%$ \\
T2K & $12 \%$ & $46 \%$ \\
NO $\nu$ A & $25 \%$ & $86 \%$ \\
\hline Combination & $9 \%$ & $42 \%$ \\
\hline
\end{tabular}

Table 2: Precision for $\left|\Delta m_{31}^{2}\right|$ and $\sin ^{2} \theta_{23}$ at $3 \sigma$ for the values $\Delta m_{31}^{2}=2 \times 10^{-3} \mathrm{eV}^{2}, \sin ^{2} \theta_{23}=0.5$.

Tab. 2 depends on the value of $\Delta m_{31}^{2}$ which is shown in fig. 22 The sensitivity suffers for all experiments for low values of $\Delta m_{31}^{2}$. T2K will provide a precise determination of $\Delta m_{31}^{2}$ at the level of a few percent for $\Delta m_{31}^{2} \gtrsim 2 \times 10^{-3} \mathrm{eV}^{2}$. Although $\mathrm{NO} \nu \mathrm{A}$ can put a comparable lower bound on $\Delta m_{31}^{2}$, the upper bound is significantly weaker, and similar to the bound from MINOS. The reason for this is a strong correlation between $\Delta m_{31}^{2}$ and $\theta_{23}$, which disappears only for $\Delta m_{31}^{2} \gtrsim 3 \times 10^{-3} \mathrm{eV}^{2}$. From the right panel of fig. 2 one can see that for $\Delta m_{31}^{2} \sim 2 \times 10^{-3} \mathrm{eV}^{2}$ only T2K is able to improve the current bound on $\sin ^{2} \theta_{23}$. One reason for the rather poor performance on $\sin ^{2} \theta_{23}$ is the fact that these experiments are sensitive mainly to $\sin ^{2} 2 \theta_{23}$. This implies that for $\theta_{23} \approx \pi / 4$ it is very hard to achieve a good accuracy on $\sin ^{2} \theta_{23}$, although $\sin ^{2} 2 \theta_{23}$ can be measured with relatively high precision [46].

$\Delta m_{31}^{2}$-precision $\quad \sin ^{2} \theta_{23}$-precision

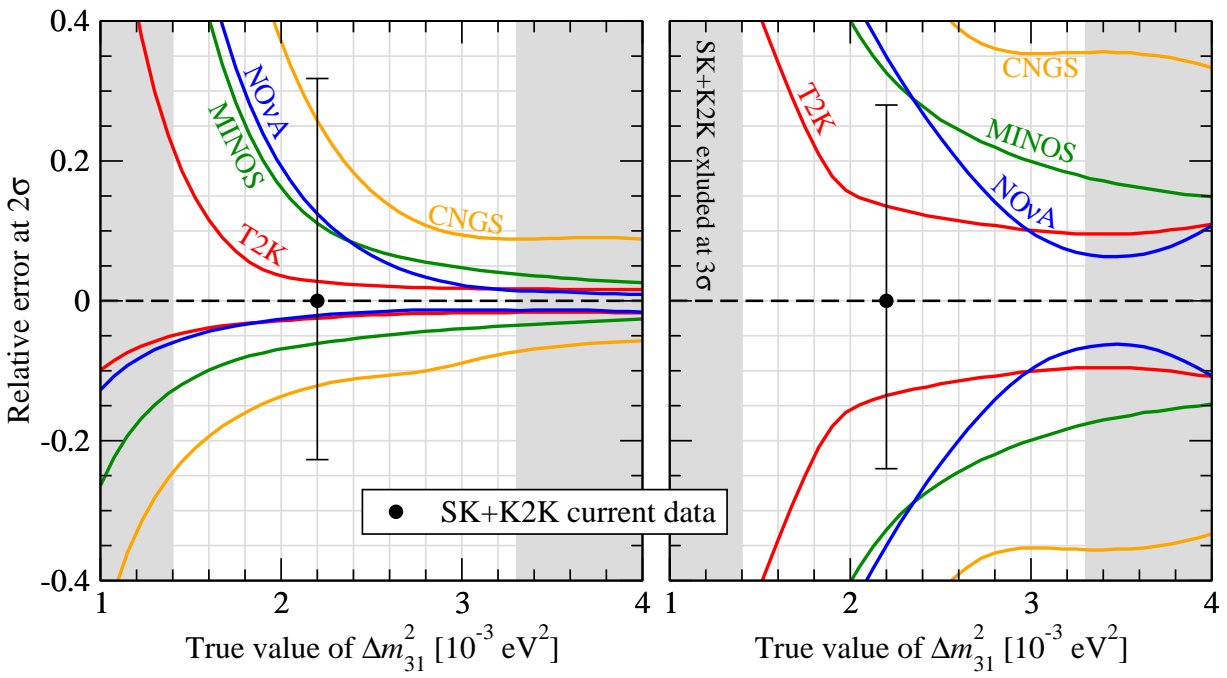

Figure 2: The precision of $\Delta m_{31}^{2}$ (left panel) and $\sin ^{2} \theta_{23}$ (right panel) as a function of the "true value" of $\Delta m_{31}^{2}$ for $\theta_{23}^{\text {true }}=\pi / 4$ (from Ref. [47]). 
Another interesting question is if the next generation long baseline experiments which will operate in the next years will be able to test the three flavourness of the oscillations. The sensitivity to a finite value of the key parameter $\theta_{13}$ is shown in fig. 3 for MINOS, OPERA and ICARUS. It can be seen that these experiments have only a modest potential for improvements of the existing $\theta_{13}$ limit.
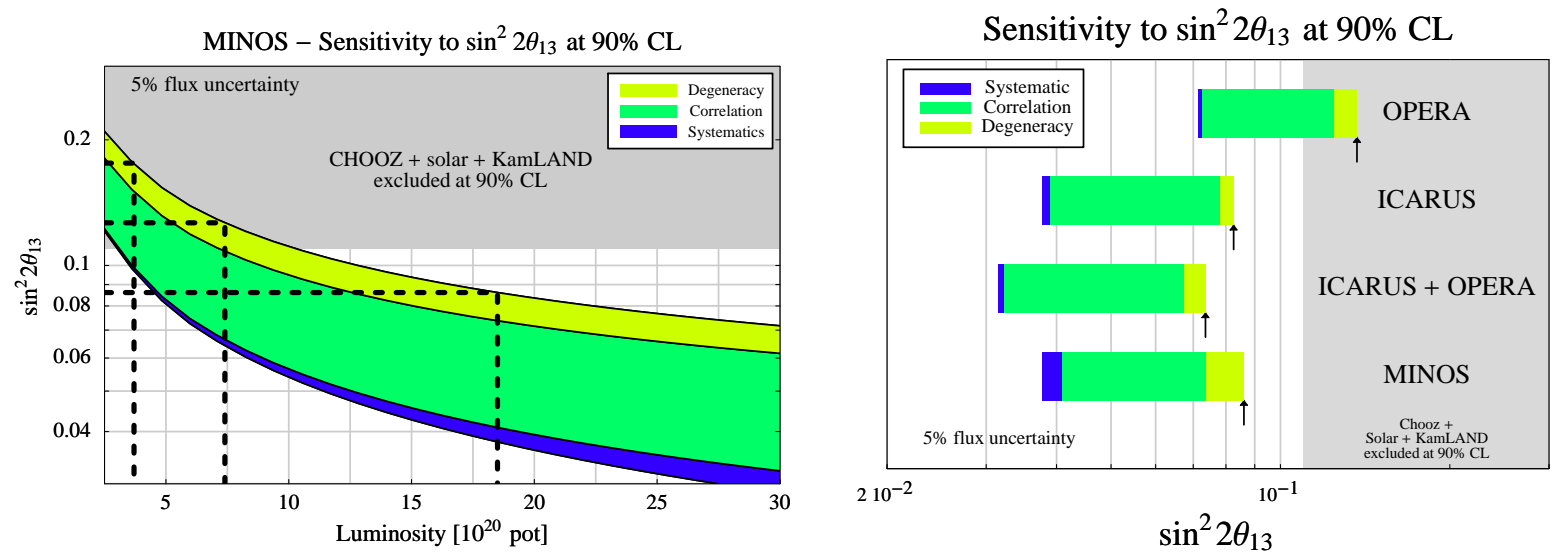

Figure 3: Left plot: The sensitivity of the MINOS experiment to $\theta_{13}$ as a function of the protons on target (pot) assuming a 5\% flux uncertainty. The dashed lines represent what 1,2 and 5 years of operation might achieve (from left to right). Right plot: Comparison of 5 years of operation for the MINOS and CNGS experiments. The grey area for large $\sin ^{2} 2 \theta_{13}$ indicates in all cases the current limit from the CHOOZ experiment. The color code of the error bars is explained in fig. 1. Further details can be found in 44.

The combined sensitivity to $\sin ^{2} 2 \theta_{13}$ of the next-to-next generation experiments is compared in the left panel of fig. 团 with new reactor experiments, T2K (JPARC-SK) and $\mathrm{NO} \nu \mathrm{A}(\mathrm{NuMI})$. It can be seen that the $\sin ^{2} 2 \theta_{13}$-limits from beam experiments are strongly affected by parameter correlations and degeneracies, whereas reactor experiments provide a "clean" measurement of $\sin ^{2} 2 \theta_{13}$, dominated by statistics and systematics [8].
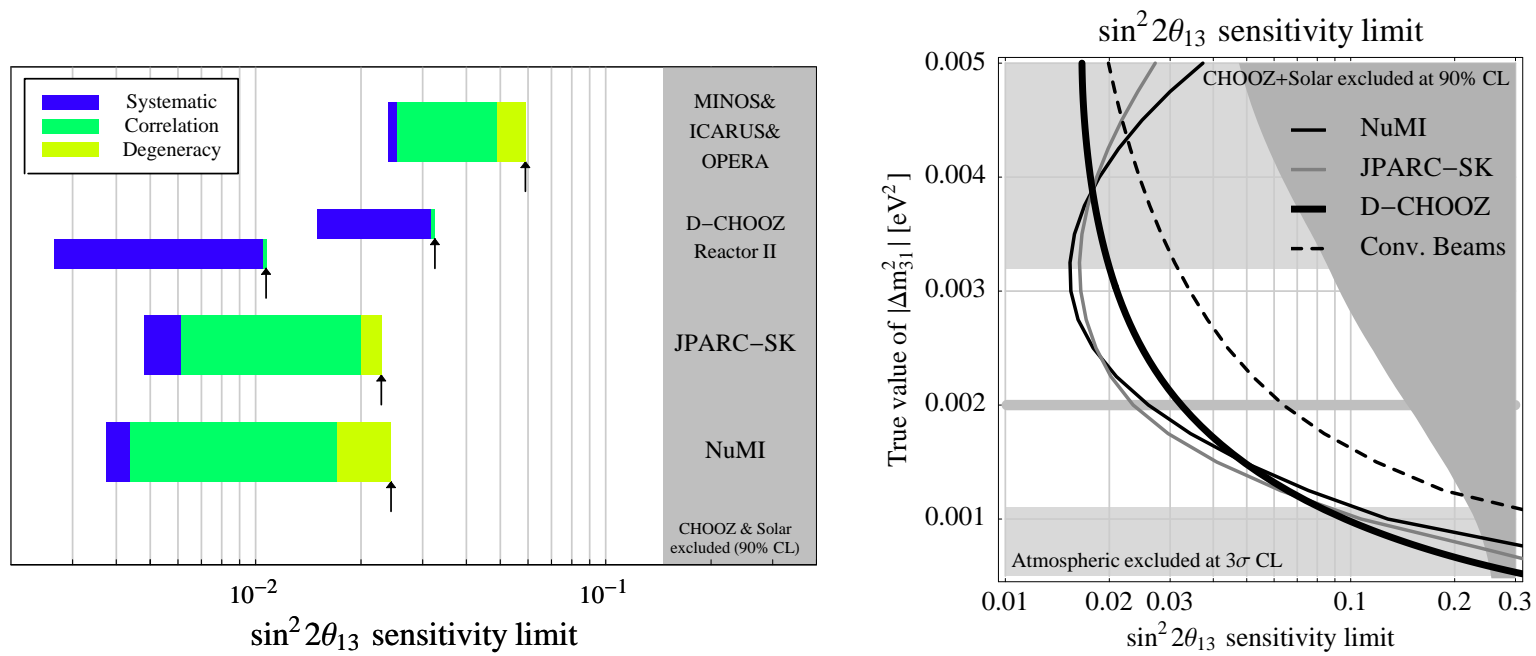

Figure 4: Left plot: Sensitivity to $\sin ^{2} 2 \theta_{13}$ at $90 \%$ CL for $\Delta m_{31}^{2}=2 \times 10^{-3} \mathrm{eV}^{2}, \Delta m_{21}^{2}=7 \times 10^{-5} \mathrm{eV}^{2}$. Right plot: Sensitivity to $\sin ^{2} 2 \theta_{13}$ at $90 \%$ CL as a function of the "true value" of $\Delta m_{31}^{2}$. 


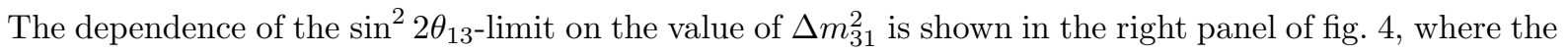
sensitivity of all experiments gets again rather poor for low values of $\Delta m_{31}^{2}$. For $\Delta m_{31}^{2} \sim 2 \times 10^{-3} \mathrm{eV}^{2}$ we find roughly an improvement by a factor 2 from conventional beam experiments (MINOS+ICARUS+OPERA combined), a factor 4 from $\mathrm{D}-\mathrm{CHOOZ}$, and a factor 6 from the superbeams $\mathrm{T} 2 \mathrm{~K}$ and $\mathrm{NO} \nu \mathrm{A}$ with respect to the current bound from global data [15]. Note that an optimized reactor experiment such as Reactor-II has the potential for even better $\sin ^{2} 2 \theta_{13}$-sensitivities than the superbeams (c.f. left panel of fig. (4).

\section{$4.2 \quad$ Synergies}

The previous discussion shows that competing plans with similar potential might be realized at the same time scale. This allows to combine the statistics of similar experiments leading to improved global fits. However, it is also possible to utilize synergies between experiments which are more than the simple addition of statistics. The point is that individual experiments measure a certain parameter combinations which contain different degeneracies and correlations. Experiments with similar sensitivities and different correlations and degeneracies allow to separate the parameters partly or fully. An example of such a discussion is given by combining the $\mathrm{T} 2 \mathrm{~K}$ and $\mathrm{NO} \nu \mathrm{A}$ experiments for a fixed time of operation in the best possible way. T2K is essentially insensitive to matter effects, while matter effects play already some role for the longer $\mathrm{NO} \nu \mathrm{A}$ baselines. Both experiments could run partly with neutrino and partly with anti-neutrino beams. The cross-sections for anti-neutrinos are, however, smaller, leading to fewer events for the same running period. An anti-neutrino running is moreover in many aspects like a different experiment, but it is clear that antineutrino information is crucial in order to resolve the parameters. A comparable reactor neutrino experiment would be very useful here. It could provide the required information such that both $\mathrm{T} 2 \mathrm{~K}$ and $\mathrm{NO} \nu \mathrm{A}$ could initially run fully in neutrino mode.

Such a synergetic combination would be especially interesting if $\sin ^{2} 2 \theta_{13}$ would be close to the current bound. In order to demonstrate these synergy effects we assume that $\sin ^{2} 2 \theta_{13}=0.1$ and investigate what we could be learned about the CP-phase $\delta$ and the neutrino mass ordering. First we note that T2K, NO $\nu \mathrm{A}$ and the reactor experiment will all be able to establish the non-zero value of $\sin ^{2} 2 \theta_{13}$. However, depending on the unknown value of $\delta$ different values of $\sin ^{2} 2 \theta_{13}$ will be allowed. This can be seen as allowed regions in the $\theta_{13}-\delta$-plane shown in Figs. 8 and 9 of Ref. [44. None of the experiments on their own can give any information on the CP-phase $\delta$ and on the mass hierarchy. The determination of $\sin ^{2} 2 \theta_{13}$ from beam experiments is strongly affected by the correlations with $\delta$, and especially for $\mathrm{NO} \nu \mathrm{A}$ also correlations with other parameters are important. Moreover, the inability to rule out the wrong mass hierarchy leads to a further ambiguity in the determination of $\sin ^{2} 2 \theta_{13}$. In contrast, since the $\bar{\nu}_{e}$-survival probability does not depend on $\delta$, Reactor-II provides a clean determination of $\sin ^{2} 2 \theta_{13}$ at the level of $20 \%$ at $90 \%$ CL. If all experiments are combined the complementarity of reactor and beam experiments allows to exclude up to $40 \%$ of all possible values of the CP-phase for a given hierarchy. The wrong hierarchy can be ruled out at a modest confidence level with $\Delta \chi^{2} \simeq 3$ due to matter effects in $\mathrm{NO} \nu \mathrm{A}$. However, at high confidence levels all values of $\delta$ are allowed, and moreover, even for a given hierarchy CP-conserving and CP-violating values of $\delta$ cannot be distinguished at $90 \%$ CL. These results depend also to some extent on the value of $\delta$. 
So far we have considered only neutrino running for the superbeams, since it is unlikely that significant data can be collected with anti-neutrinos within ten years from now. Nevertheless, it might be interesting to investigate the potential of a neutrino-antineutrino comparison. In fig. 5 we show the results from $\mathrm{T} 2 \mathrm{~K}+\mathrm{NO} \nu \mathrm{A}$ with 3 years of neutrinos plus 3 years of anti-neutrinos each (left), in comparison with the case where the antineutrino running is replaced by Reactor-II (right). We find that antineutrino data at that level does neither solve the problems related to the CP-phase nor to the hierarchy. Still CP-violating and CP-conserving values cannot be distinguished at 90\% CL. Moreover, the determination of $\sin ^{2} 2 \theta_{13}$ is less precise than from the reactor measurement. To benefit from antineutrino measurements a significantly longer measurement period would be necessary, to obtain large enough data samples.

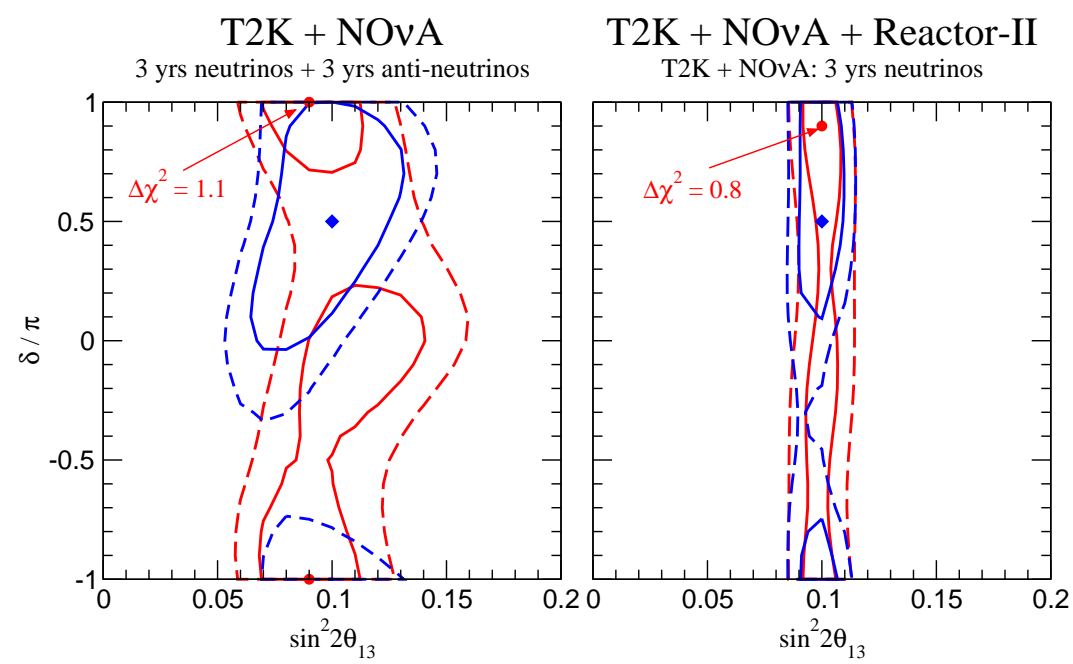

Figure 5: Antineutrino running vs Reactor-II. We show the $90 \%$ CL (solid curves) and $3 \sigma$ (dashed curves) allowed regions in the $\sin ^{2} 2 \theta_{13}-\delta$-plane for the assumed values $\sin ^{2} 2 \theta_{13}=0.1$ and $\delta=\pi / 2$. The blue/dark curves refer to the allowed regions for the normal mass hierarchy, whereas the red/light curves refer to the $\operatorname{sgn}\left(\Delta m_{31}^{2}\right)$-degenerate solution (inverted hierarchy), where the projections of the minima onto the $\sin ^{2} 2 \theta_{13}-\delta$ plane are shown as diamonds (normal hierarchy) and dots (inverted hierarchy). For the latter, the $\Delta \chi^{2}$-value with respect to the best-fit point is also given.

\subsection{Long term perspectives}

Beyond the discussed accelerator and reactor based oscillation experiments exist more ambitious projects like the JHF-HyperKamiokande project, beta beams and neutrino factories. Such experiments clearly require further R\&D before they can be built. However, assuming current knowledge, we believe that such setups are possible in the long run. The potential of the JHF-HyperKamiokande experiment and a neutrino factory are compared in fig. [6] to T2K (JHF-SK) and $\mathrm{NO} \nu \mathrm{A}(\mathrm{NuMI})$. It can be seen that the existing limits can be improved by a few orders of magnitude compared to now.

\section{Theoretical implications and conclusions}

One of the most interesting unsolved topics is the origin of flavour and fermion masses. There exist apparent regularities in the fermionic field content which make it very tempting to introduce right-handed neutrino 


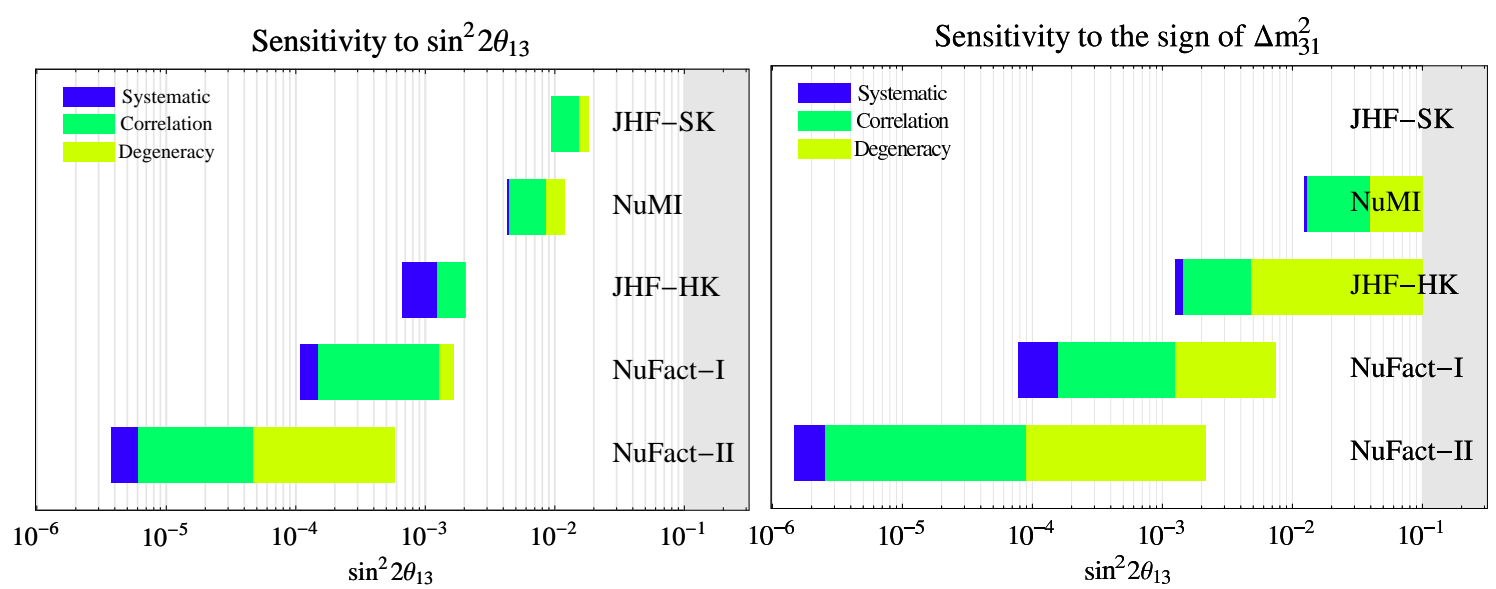

Figure 6: Left plot: The $\theta_{13}$ sensitivity of different future accelerator based neutrino oscillation experiments 20]. Right plot: The $\theta_{13}$ values for which sensitivity to matter effects, i.e. $\operatorname{sign}\left(\Delta m_{31}^{2}\right)$ exists. The shown bands are again the reduction of sensitivity from a purely statistical limit (left end of the dark grey/blue range) by systematics (right end of dark grey/blue), correlations (medium grey/green) and degeneracies (light grey/yellow). The right end of the light grey(yellow) band represents the final 90\%CL limit. The grey area for large $\sin ^{2} 2 \theta_{13}$ indicates the current limit from the CHOOZ experiment.

fields leading to both Dirac and Majorana mass terms for neutrinos. Diagonalization of the resulting mass matrices yields Majorana mass eigenstates and due to the see-saw mechanism very small neutrino masses. This can also be nicely realized in embeddings of the SM into GUTs with larger symmetries, such as $\mathrm{SO}(10)$. Before the discovery of large leptonic mixing, many theorists expected the leptonic mixings to be similar to quark mixing, characterized by small mixing angles. Experiment led theory in showing the striking results that $\sin ^{2} 2 \theta_{23} \simeq 1$ and $\tan ^{2} \theta_{12} \simeq 0.39$, while $\theta_{13}$ is small. By finding two large mixing angles, neutrino physics has already provided surprising and very valuable information which severely constrains models of neutrino masses. Future precision neutrino oscillation experiments will provide further precision tests of the flavour sector. The level of precision will confirm or rule out ideas about the origin of flavour and connected topics.

An important subject is the small value of $\sin ^{2} 2 \theta_{13}$. Since there are two large mixing angles, there is no particular reason to expect the third angle, $\theta_{13}$, to be extremely small or even zero. A small value of $\sin ^{2} 2 \theta_{13} \simeq 0.1$ could be a numerical coincidence in a framework which predicts generically large or sizable mixings. However, if the limit on $\sin ^{2} 2 \theta_{13}$ would become smaller by an order of magnitude then some protective mechanism like a "symmetry" would be required. This can be seen in neutrino mass models which are able to predict the large values for $\theta_{12}$ and $\theta_{23}$. Such models have a certain tendency to predict a sizable value of $\theta_{13}$ as can be seen, for example, in [49, 50, 51, 52, 53, 54. The conclusion is that a value of $\theta_{13}$ close to the CHOOZ bound would be quite natural, while much smaller values are less likely or hard to understand.

Future precision measurements can also test if relations like $\theta_{23}=\pi / 4$ [55] or $\theta_{12}+\theta_{C}=\pi / 4$ which are currently fulfilled within experimental errors still hold at much better precision. If so, then this would provide strong constrains on the origin of the flavour structure. Precision is also valuable, even if such special relations are not found. The point is that it is generally not easy to predict a set of very precisely known 
masses and mixings in a certain model or class of models.

Neutrino masses and mixing parameters are also subject to quantum corrections between low scales, where measurements are performed, and high scales where some theory typically predicts the masses and mixings. This has interesting implications, since it implies that certain deviations from special relations are expected due to quantum corrections (renormalization group or RGE effects). Suppose, for example, that some theory were able to predict $\theta_{13} \equiv 0$. Then RGE effects would still predict a tiny, but finite value at low

energy. Strictly speaking, $\theta_{13}=0$ cannot be excluded completely by this argument, as the high-energy value could be just as large as the change due to running and of opposite sign. However, a complete cancellation of this kind would be a miraculous fine-tuning, since the physics generating the value at high energy is not directly related to quantum corrections at lower energies. The strength of the running of $\theta_{13}$ depends on the neutrino mass spectrum and whether or not supersymmetry is realized. For the Minimal Supersymmetric Standard Model one finds a shift $\Delta \sin ^{2} 2 \theta_{13}>0.01$ for a considerable parameter range, i.e. one would expect to measure a finite value of $\theta_{13}$ [56]. Conversely, limits on model parameters would be obtained if an experiment were to set an upper bound on $\sin ^{2} 2 \theta_{13}$ in the range of 0.01 . In any case, it should be clear that a precision of the order of quantum corrections to neutrino masses and mixings is very interesting in a number of ways.

Precision measurements would also allow very interesting tests of many other topics, like MSW matter effects, three neutrino unitarity, neutrino decay, de-coherence and NSI effects. There exists also an interesting interplay with theories beyond the Standard Models, flavour models as well as astroparticle physics (leptogenesis, supernovae, nucleosynthesis, structure formation). In summary, future precision oscillation experiments will provide the best window into the so far un-understood flavour sector. It may give us a glimpse on the origin of flavour, but it may also lead to unexpected results and insights as it happened before in neutrino physics.

Acknowledgments: Work supported by the "Sonderforschungsbereich 375 für Astro-Teilchenphysik der Deutschen Forschungsgemeinschaft".

\section{References}

[1] T. Toshito (SuperKamiokande Collab.), proceedings of 36th Rencontres de Moriond on Electroweak Interactions and Unified Theories, Les Arcs, France, 10-17 Mar 2001, hep-ex/0105023

[2] W. Hampel et al. (GALLEX Collab.), Phys. Lett. B 447 (1999) 127.

[3] A. McDonald, these proceedings.

[4] Q. R. Ahmad et al. (SNO Collab.), Phys. Rev. Lett. 89 (2002) 011301, nucl-ex/0204008.

[5] A. Suzuki, these proceedings.

[6] K. Eguchi et al. (KamLAND Collab.), Phys. Rev. Lett. 90 (2003) 021802, hep-ex/0212021.

[7] E.D. Church, K. Eitel, G.B. Mills and M. Steidl, Phys. Rev. D 66 (2002) 013001, hep-ex/0203023. 
[8] Y. Suzuki, these proceedings.

[9] M.C. Gonzalez-Garcia, these proceedings.

[10] S. Fukuda et al., Phys. Lett. B 539 (2002) 179; B.T. Cleveland et al., Astrophys. J. 496(1998) 505; D.N. Abdurashitov et al., Phys. Rev. C60 (1999) 055801; W. Hampel et al., Phys. Lett. B447 (1999) 127; C. Cattadori, Nucl. Phys. B (Proc. Suppl.) 110 (2002) 311; Q. R. Ahmad et al., Phys. Rev. Lett. 89 (2002) 011301; Phys. Rev. Lett. 89 (2002) 011302.

[11] SNO collaboration, submitted to Phys. Rev. Lett. 2003, nucl-ex/0309004.

[12] M. Apollonio et al., Phys. Lett. B 420 (1998) 397.

[13] M. Apollonio et al., Eur. Phys. J. C 27 (2003) 331.

[14] F. Boehm et al., Phys. Rev. Lett. 84 (2000) 3764.

[15] M. Maltoni, T. Schwetz, M.A. Tortola and J.W.F. Valle, New Jour. Phys. 6, (2004) 122, hep-ph/0405172 Phys. Rev. D 68, (2003) 113010, hep-ph/0309130

[16] M. Freund, P. Huber and M. Lindner, Nucl. Phys. B 585 (2000) 105, hep-ph/0004085.

[17] M. Freund, P. Huber and M. Lindner, Nucl. Phys. B 615 (2001) 331, hep-ph/0105071.

[18] P. Huber, M. Lindner and W. Winter, Nucl. Phys. B 654 (2003) 3, hep-ph/0211300.

[19] M. Lindner, in 'Neutrino mass', Springer tracts in modern physics, ed. by G. Altarelli and K. Winter, hep-ph/0209083.

[20] P. Huber, M. Lindner and W. Winter, Nucl. Phys. B 645 (2002) 3, hep-ph/0204352.

[21] K. Dick, M. Freund, M. Lindner and A. Romanino, Nucl. Phys. B 562 (1999) 29, hep-ph/9903308.

[22] M. Freund, Phys. Rev. D64 (2001) 053003, hep-ph/0103300.

[23] E. K. Akhmedov, R. Johansson, M. Lindner, T. Ohlsson and T. Schwetz, JHEP 0404 (2004) 078, hep-ph/0402175

[24] A. Cervera et al., Nucl. Phys. B579 (2000) 17, erratum ibid., Nucl. Phys. B593, (2001) 731.

[25] H. Minakata, H. Sugiyama, O. Yasuda, K. Inoue and F. Suekane, Phys. Rev. D 68 (2003) 033017, hep-ph/0211111.

[26] P. Huber, M. Lindner, T. Schwetz and W. Winter, Nucl. Phys. B 665 (2003) 487, hep-ph/0303232.

[27] P. Huber and W. Winter, Phys. Rev. D 68 (2003) 037301, hep-ph/0301257

[28] G. L. Fogli and E. Lisi, Phys. Rev. D 54 (1996) 3667, hep-ph/9604415.

[29] V. Barger, D. Marfatia, and K. Whisnant, Phys. Rev. D65 (2002) 073023, hep-ph/0112119. 
[30] M. Koike, T. Ota and J. Sato, Phys. Rev. D 65 (2002) 053015, hep-ph/0011387.

[31] J. Burguet-Castell, M.B. Gavela, J.J. Gomez-Cadenas, P. Hernandez and O. Mena, Nucl. Phys. B608 (2001) 301, hep-ph/0103258.

[32] H. Minakata and H. Nunokawa, JHEP 10 (2001) 001, hep-ph/0108085.

[33] J. Burguet-Castell, M.B. Gavela, J.J. Gomez-Cadenas, P. Hernandez and O. Mena, Nucl. Phys. B 646 (2002) 301, hep-ph/0207080.

[34] V. Barger, D. Marfatia and K. Whisnant, Phys.Rev. D66 (2002) 053007, hep-ph/0206038.

[35] P. Huber, M. Maltoni and T. Schwetz, hep-ph/0501037.

[36] A. Donini, D. Meloni and P. Migliozzi, Nucl.Phys. B 646 (2002) 321, hep-ph/0206034.

[37] E. Ables et al. (MINOS) FERMILAB-PROPOSAL-P-875.

[38] P. Aprili et al. (ICARUS) CERN-SPSC-2002-027.

[39] D. Duchesneau (OPERA), hep-ex/0209082.

[40] Y. Itow et al., hep-ex/0106019.

[41] D. Ayres et al., hep-ex/0210005,

[42] K. Anderson et al., hep-ex/0402041

[43] F. Ardellier et al., hep-ex/0405032

[44] P. Huber, M. Lindner, M. Rolinec, T. Schwetz and W. Winter, Phys. Rev. D 70 (2004) 073014, hep-ph/0403068.

[45] P. Huber, M. Lindner and W. Winter, GLoBES (Global Long Baseline Experiment Simulator), hep-ph/0407333, to appear in Comp. Phys. Comm., http://www.ph.tum.de/ globes.

[46] H. Minakata, M. Sonoyama and H. Sugiyama, Phys. Rev. D 70 (2004) 113012, hep-ph/0406073

[47] P. Huber, M. Lindner, M. Rolinec, T. Schwetz and W. Winter, hep-ph/0412133

[48] P. Huber, M. Lindner, T. Schwetz and W. Winter, Nucl. Phys. B 665 (2003) 487, hep-ph/0303232

[49] S. M. Barr and I. Dorsner, Nucl. Phys. B 585 (2000) 79, hep-ph/0003058.

[50] G. Altarelli and F. Feruglio, in 'Neutrino Mass', Springer Tracts in Modern Physics, ed. by G. Altarelli and K. Winter, hep-ph/0206077.

[51] R. Barbieri, T. Hambye and A. Romanino, JHEP 0303 (2003) 017, hep-ph/0302118.

[52] M. C. Chen and K. T. Mahanthappa, Int.J.Mod.Phys. A18 (2003) 5819, hep-ph/0305088. 
[53] S. F. King, Rept. Prog. Phys. 67 (2004) 107, hep-ph/0310204

[54] H. S. Goh, R. N. Mohapatra and S. P. Ng, Phys. Rev. D 68 (2003) 115008, hep-ph/0308197

[55] S. Antusch, P. Huber, J. Kersten, T. Schwetz and W. Winter, Phys. Rev. D 70 (2004) 097302, hep-ph/0404268

[56] S. Antusch, J. Kersten, M. Lindner and M. Ratz, Nucl. Phys. B 674 (2003) 401, hep-ph/0305273. 